\title{
Implementation of an Autonomous Multi-Maneuver Targeting Sequence for Lunar Trans-Earth Injection
}

\author{
Ryan J. Whitley ${ }^{1}$ and Jacob Williams ${ }^{2}$ \\ NASA Johnson Space Center, Houston, TX, 77058
}

\begin{abstract}
Using a fully analytic initial guess estimate as a first iterate, a targeting procedure that constructs a flyable burn maneuver sequence to transfer a spacecraft from any closed Moon orbit to a desired Earth entry state is developed and implemented. The algorithm is built to support the need for an anytime abort capability for Orion.
\end{abstract}

\section{Introduction}

$\mathrm{B}$ ASED on project requirements, the Orion spacecraft must be able to autonomously calculate the translational maneuver targets for an entire Lunar mission. Translational maneuver target sequences for the Orion spacecraft include Lunar Orbit Insertion (LOI), Trans-Earth Injection (TEI), and Trajectory Correction Maneuvers (TCMs). This onboard capability is generally assumed to be supplemental to redundant ground computation in nominal mission operations and considered as a viable alternative primarily in loss of communications contingencies.

Of these maneuvers, the ability to accurately and consistently establish a flyable 3-burn TEI target sequence is especially critical. The TEI is the sole means by which the crew can successfully return from the Moon to a narrowly banded Earth Entry Interface (EI) state. This is made even more critical by the desire for global access on the lunar surface. Currently, the designed propellant load is based on fully optimized TEI solutions for the worst case geometries associated with the accepted range of epochs and landing sites. This presents two challenges for an autonomous algorithm: in addition to being feasible, the targets must include burn sequences that do not exceed the anticipated propellant load.

\section{Algorithm Design}

In this paper, an adaptive targeting algorithm that merges an initial guess procedure with a robust iterative nonlinear solver is presented. This algorithm has flexible application to either ground based targeting and/or onboard computation as it is designed to converge on solutions quickly and robustly. Since a fully integrated solution is required that handles third body effects which cannot be solved for analytically, a patched conic method has been developed to establish the initial guess. ${ }^{1}$ At this point an iterative scheme that minimizes the discrepancy in the states of the trajectory is necessary.

The Trans-Earth Injection (TEI) sequence has a general design of three consecutive burns due to the significant cost savings that exists over a single burn in the full range of anticipated mission scenarios. While both single and three burn solutions can be obtained, the total cost of a three burn sequence is almost always less expensive. This is especially true when the geometry requires a large plane change from the initial Moon orbit to the Earth-Moon transfer arc and is used to determine propellant loads. Regardless of the date or orbit, the three burn sequence has the same basic structure. The first burn significantly raises the apolune of the Low Lunar Orbit (LLO). This is generally the largest maneuver of the three conducted in the plane of the initial Moon orbit. The second burn occurs at or near to the apolune of this new large elliptical orbit and places the spacecraft in the departure plane. The cost to change the direction of the velocity vector is cheapest when the velocity magnitude is at a minimum. This is generally the least expensive maneuver. The third and final burn occurs when the spacecraft sweeps back towards the Moon near perilune. This final burn places the spacecraft on a very specific trajectory designed to hit a particular entry interface (EI) condition that can, depending on the landing site, have very tight constraints. Parameters involved in the selection of a viable EI state include altitude, flight path angle, azimuth, longitude, and latitude targets.

Flexibility to accurately set up targets for the full range of epochs and orbits autonomously is an important attribute of the algorithm. To that end, a vector based approach is used that purposely avoids the use of complex trigonometric expressions due to their intrinsic singularities. As such, vector equations have been derived to compute an associated $\mathrm{v}_{\infty}$ vector for an Earth return trajectory analytically. This vector is determined by the desired

\footnotetext{
${ }^{1}$ Aerospace Engineer, Orion GN\&C, NASA Johnson Space Center, EG5, AIAA Member

${ }^{2}$ Aerospace Engineer, Orion GN\&C, ESCG, AIAA Member
} 
flight time and calculated from the resultant transfer arc between the current Moon state and associated antipode state at the Earth. ${ }^{1}$

Targeting a $\mathrm{v}_{\infty}$ vector is different from successfully reaching a specific EI state. Over a multiple day transfer the impact of Earth and Sun gravitational perturbations can invoke large changes in the final state at the Earth leading the spacecraft far from the desired EI targets. But to correct these discrepancies, instead of propagating forward to EI, the optimization parameters associated with the target Earth state are propagated backwards in time. Setting a single point to match position and velocity for a continuous trajectory in the Moon sphere of influence (SOI) reduces the convergence difficulties associated with the large swings in EI parameters that correspond to small changes near the Moon.

The desire for a flexible autonomous algorithm is naturally coupled with a need to minimize excessive iteration. Once the analytic guess is determined, it is vital that the targeter quickly converge to a feasible, flyable solution. There exists a variety of algorithms that take advantage of the assumption that the solution lies within the vicinity of the initial guess by designing search directions for the variable control parameters that point towards the minimum feasible solution as efficiently as possible. The TEI autonomous targeter takes advantage of two of these techniques. One option is a full optimization mode, where the constraints and total fuel consumption objective function are simultaneously minimized. A Sequential Quadratic Program (SQP) is selected as the algorithm for full optimization due to the quadratic nature of the solution space where the initial guess is in the vicinity of the solution. This option requires more iteration than the second option, which is a minimum norm mode. The minimum norm algorithm does not attempt to minimize the solution but instead chooses a search direction based that minimizes the constraint error. This correction is calculated using the simple pseudo-inverse mathematical operation. Without a $\Delta \mathrm{v}$ constraint, this approach always converges robustly close to the fuel cost of the initial guess. If a mission cost constraint is added in the form of a maximum quantity of fuel ( $\Delta \mathrm{v}$ limit), convergence is still robust, but careful attention must be made to the constraint value used. The optimal value in a given Epoch and geometry is not known apriori and if the limit is too constraining the algorithm could be set up to solve a problem in which no solution exists. As long as the propellant load exceeds performance estimates, this value could be set to the propellant limit, assuming margin.

Once a converged impulsive solution has been obtained, the targeting algorithm can convert each individual $\Delta \mathrm{v}$ into a finite burn command. A two point boundary value problem is setup where the number of controls and constraints are equal. Using a velocity direction based spherical formulation of the burn a simple non-linear solver may be used. The minimum norm algorithm converges these finite burn conversions robustly, even for low thrust engines.

\section{References}

${ }^{1}$ Saudemont, R. R. and Cesar A. Ocampo. "Initial Trajectory Model for a Multi-Maneuver Moon to Earth Abort Sequence," AAS Space Flight Mechanics Meeting, 2009. 


\title{
Implementation of an Autonomous Multi-Maneuver Targeting Sequence for Lunar Trans-Earth Injection
}

\author{
Ryan J. Whitley ${ }^{*}$, Cesar A. Ocampo ${ }^{\dagger}$ and Jacob Williams ${ }^{\ddagger}$ \\ NASA Johnson Space Center, Houston, TX, 77058
}

\begin{abstract}
Using a fully analytic initial guess estimate, a targeting procedure that constructs a flyable multi-burn maneuver sequence to transfer a spacecraft from any closed Moon orbit to a desired Earth entry state is developed and implemented. The algorithm is built to support the need for an anytime abort capability for the Orion spacecraft.
\end{abstract}

\section{Introduction}

$\mathrm{B}$ ASED on project requirements, the Orion spacecraft must be able to autonomously calculate the translational maneuver targets for an entire Lunar mission. Translational maneuver target sequences for the Orion spacecraft include Lunar Orbit Insertion (LOI), Trans-Earth Injection (TEI), and Trajectory Correction Maneuvers (TCMs). This onboard capability is generally assumed to be supplemental to redundant ground computation in nominal mission operations and considered as a viable alternative primarily in loss of communications contingencies.

Of these maneuvers, the ability to accurately and consistently establish a flyable 3-burn TEI target sequence is especially critical. The TEI is the sole means by which the crew can successfully return from the Moon to a narrowly banded Earth Entry Interface (EI) state. This is made even more critical by the desire for global access of the lunar surface. Currently, the designed propellant load is based on fully optimized TEI solutions for the worst case geometries associated with the accepted range of epochs and landing sites ${ }^{1}$. This presents two challenges for an autonomous algorithm: in addition to being feasible, the targets must include burn sequences that do not exceed the anticipated propellant load.

The Trans-Earth Injection (TEI) sequence has a general design of three burns due to the significant cost savings that exists over a single burn in the full range of anticipated mission scenarios. While both single and three burn solutions can be obtained at all times, the total cost of a three burn sequence is almost always less expensive. This is especially true when the geometry requires a large plane change from the initial Moon orbit to the Earth-Moon transfer arc. Regardless of the date or orbit, the three burn sequence has the same basic structure. The first burn significantly raises the apolune of the Low Lunar Orbit (LLO). This is generally the largest maneuver of the three conducted in or close to the plane of the initial Moon orbit. The second burn occurs near the apolune of this first transfer orbit and places the spacecraft in the departure plane. The cost to change the direction of the velocity vector is cheapest when the velocity magnitude is at a minimum. This is generally the least expensive maneuver. The third and final burn occurs when the spacecraft sweeps back towards the Moon near perilune. This final burn places the spacecraft on an explicit trajectory designed to achieve a particular entry interface (EI) condition with specified constraints. An EI state designed to deliver Orion to a specific location includes a correlated combination of altitude, flight path angle, azimuth, longitude, and latitude constraints.

\section{Algorithm Architecture: Framework for Problem Solving}

In this paper, an adaptive targeting algorithm that merges an initial guess procedure with a robust iterative nonlinear solver is presented. This algorithm has flexible application to either ground based targeting and/or onboard computation as it is designed to converge on solutions quickly and robustly. Since a fully integrated solution is required that handles third body effects which cannot be solved for analytically, a patched conic method has been developed to establish the initial guess. ${ }^{2}$ At this point an iterative scheme that minimizes the discrepancy between the states of the trajectory is necessary. Iterates that require fewer operations are desirable; thus, the solution is solved first impulsively and then each burn is converted individually into finite burn arcs. This conversion process is detailed in section VI.

\footnotetext{
* Aerospace Engineer, Orion GN\&C, NASA Johnson Space Center, EG5, AIAA Member

${ }^{\dagger}$ Associate Professor, Department of Aerospace Engineering and Engineering Mechanics, The University of Texas at Austin, AIAA Member.

${ }^{\ddagger}$ Aerospace Engineer, Orion GN\&C, ERC Inc., Engineering and Science Contract Group, AIAA Member
} 


\section{A. Fully Analytic Initial Guess}

Flexibility to accurately set up targets for the full range of epochs and orbits autonomously is an important attribute of the algorithm. To that end, a vector based approach is used that purposely avoids the use of complex trigonometric expressions due to their intrinsic singularities. As such, vector equations have been derived to compute an associated $\mathrm{v}_{\infty}$ vector for an Earth return trajectory analytically. A detailed description of the procedure to construct a first iterate patched conic solution for either a one or three burn TEI sequence has been published. ${ }^{2}$ The inputs are simple: the initial moon orbit in Keplerian parameters, an associated epoch, and a final Earth state. The final Earth state may be a set of complex correlated EI constraints, but the initial guess requires only a radial and latitude target to establish the $\mathrm{v}_{\infty}$ vector estimate. This vector is determined by the desired flight time and calculated from the resultant Lambert transfer arc between the current Moon orbit and epoch dependent antipode state at the Earth.

Regardless of the number of burns, the departure angular momentum vector is determined automatically by the intersection of the lunar parking orbit and the plane of the departure hyperbola. For the one burn sequence there is one exact hyperbola that contains the maneuver vector perpendicular to the angular momentum $/ \mathrm{v}_{\infty}$ plane, which is used to fix the time and magnitude of the impulsive estimate. In contrast, the three burn sequence setup has many more control variables at its disposal and requires additional assumptions that bound the intermediate transfer orbits. The first assumption establishes the orbit planes. As described in the introduction, it is known that the plane change is conducted primarily by the second maneuver in an optimal sequence. This fact is applied to the initial guess by constraining the original parking and first transfer ellipse orbits to be coplanar as well as the second transfer ellipse and departure hyperbola orbits. The second assumption establishes the orientation of the transfer ellipses. In the original formulation, this is set by aligning the apsides of the departure hyperbola and the second transfer ellipse. This particular underlying assumption is a key factor in determining the initidik cost and can be lowered by applying additional knowledge about the optimal solution, as described in Section IV.

\section{B. Determining the Number of Burns}

The third body perturbation effects of the Earth and the Sun are not taken into account in the formulation of the initial guess. This is for a good reason; there are no analytic models available for the complex interaction of multiple bodies, unless simplifying assumptions are applied. As a result, it is difficult to determine the optimal number of burns in the departure sequence. Researchers have attempted to establish a switching criterion for the elliptical to hyperbolic asymptote problem. One such switching criterion allows a differentiation between three impulses and four impulses, but the cost savings is negligible. ${ }^{3}$ In addition, the parametric does not extend to solving a problem with third body effects. The most useful criterion is to make use of the relative declination, an assessment of the quantity of plane change necessary in a given TEI sequence design. As the parking orbit is constant, the $\mathrm{v}_{\infty}$ vector alone determines the magnitude of this parameter. Looking at optimal results, it can be seen that for cases with relative declination quantities less than or equal to about 5 degrees (the limit varies based on the intermediate orbits), the one burn sequence is preferable. However, the optimal solution produces an unknown delta to the $\mathrm{v}_{\infty}$ vector, which results in a different relative declination from that calculated in the initial guess.

If the relative declination change that occurs during the optimization process can be predicted, an apriori switching criterion could possibly be developed. Until that time, it is recommended to calculate both one and three burn solutions and to either select the cheapest, or the simplest within propellant limits. It can be quickly seen from analysis that unless a Moon parking orbit is designed always to be closely aligned with the departure plane, or a generous propellant budget is planned, a three burn sequence will almost surely be required to return to Earth.

\section{Targeting Earth Interface (EI) Constraints}

A significant part of a successful TEI sequence is a successful Earth reentry. The transfer is not over when a spacecraft reaches EI, the point at which the atmosphere begins and the transfer trajectory ends. Depending on the properties of the spacecraft, including fixed design parameters such as ballistic number and lift-to-drag ratio, there can be little allowable deviation in the conditions at which a successful entry can occur. For any Orion entry, guided or ballistic, a narrow flight path angle corridor and velocity limit must be met. Fortunately, the excessive heat associated with a Lunar entry is designed with margin, so typically a constraint is not applied to the relative velocity magnitude. Flight path angle, on the other hand, must be within a corridor that can be less than a single degree wide or the spacecraft will either burn up or skip out. Along the way back to the Earth, the Trajectory Correction Maneuvers (TCMs) may be used to re-target the spacecraft to reach a desired state, but this capability is limited and becomes even more inadequate as the spacecraft moves closer to the Earth. The most basic EI condition is an 
altitude/flight path angle coupled constraint and is the minimum required target constraint in the TEI problem if there is any expectation of a successful entry, descent and landing (EDL).

For Orion, a specific water landing site is desired. This necessarily complicates the EI constraints. The ability to hit a specific water coastal landing site depends on the type of entry guidance employed; for Orion, two types of guided entries are considered: skip and direct. Translating into orbit terms skip means a longer range from EI to landing, and direct means a shorter range. Both have cross-range requirements. When converted to an Earth coordinate system, what results is a map of correlated latitude, longitude and azimuth constraints with range boundaries and service module disposal cutouts. The Orion entry GN\&C team maintains this database of the acceptable EI points at discrete intervals. Plotted on a map of the Earth it appears as an irregular mass of points, but while the latitude and longitude points are not continuous, the relationship between them and azimuth correlates in a measurable fashion. The reason why a set of EI points is needed is that on a given day from a given orbit, not every point is a feasible target from the Moon, much less optimal. This is in part determined by the latitude of the EarthMoon antipode. With this information, a huge swath of the EI map can be eliminated. For the targeter, this means that only a certain small band of latitude cases should be considered or iterated on. Modes are available in which the EI parameters are constrained to fixed values or boundaries (formed as inequality constraints), but care needs to be made in applying these constraints as the problem could be constrained so that no solution exists.

With the complicated shape of the EI map and the third body perturbations, it is difficult to strictly determine an appropriate latitude band, although some research has been done on the use of inequality constraints on longitude, latitude and azimuth to determine an optimal EI target state ${ }^{4}$. As a result, two multi-degree polynomial functions were generated with longitude and azimuth each as a function of latitude. Figure 1 shows the target line associated with a water landing off of the coast of San Clemente in California.

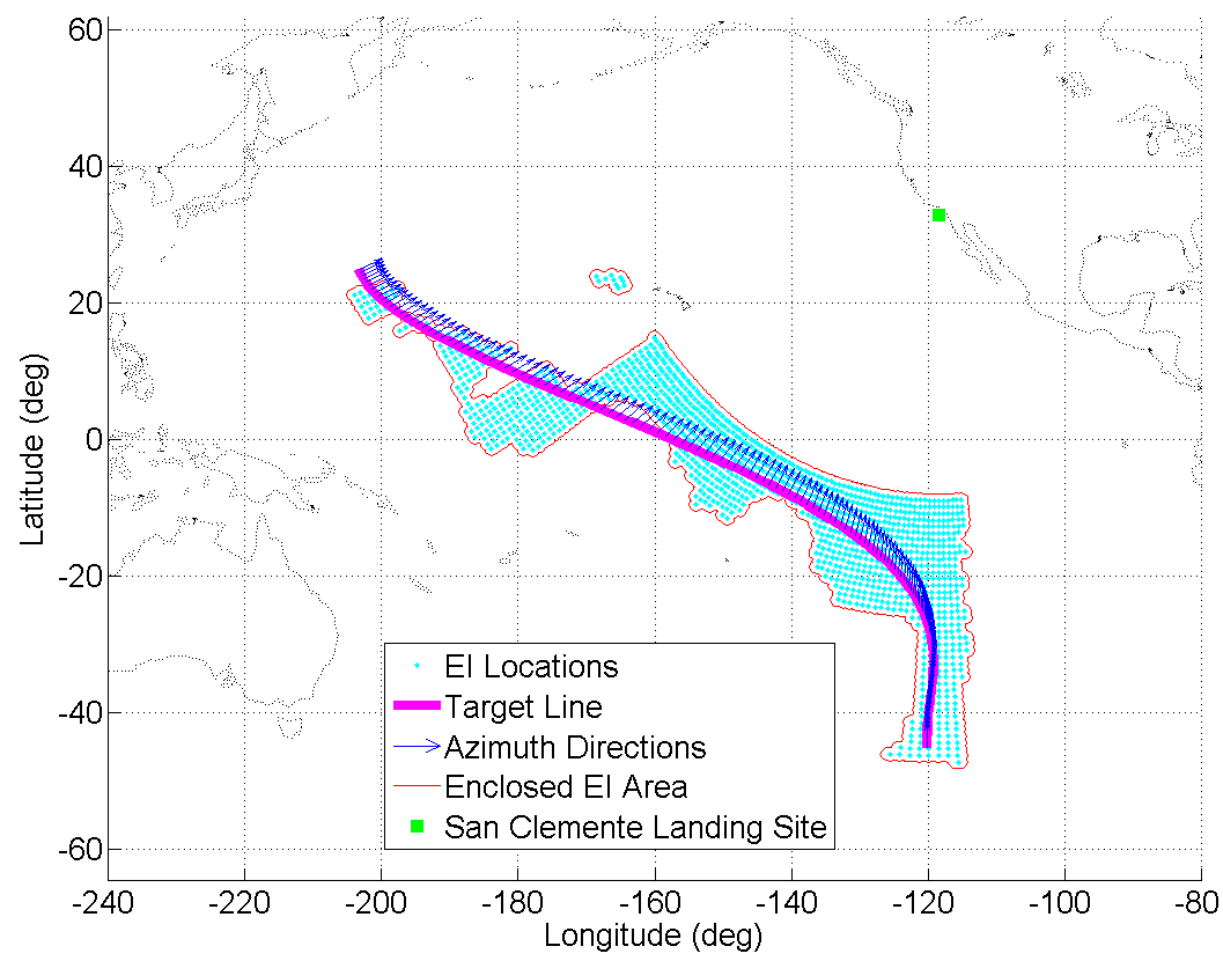

Figure 1. EI target line for San Clemente landing site.

For a free time problem, longitude can be adjusted by simply adding or subtracting mission time and thus latitude is only constrained through the longitude and azimuth functions. The target lines are drawn so that a full swath of possible latitudes is represented monotonically increasing as longitude proceeds westward and azimuth increases from zero. The continuous polynomial equations that make up the target lines are easily iterated on to obtain either feasible or optimal solutions. The EI targets are obviously tied directly to the EI map. Thus, the algorithm is free to select any user specified pre-tabulated curve tied explicitly to a desired landing site. 


\section{Gradient Determination: Minimizing Numerical Errors}

None of the tools used to solve non-linear problems (including optimizers, boundary value problems solvers and minimum norm solvers) can prove analytically that they will achieve convergence. Thus, any level of claimed convergence guarantee is solely validated through simulation and testing. The results in Section VII highlight the progress on this front. To that end, all potential stumbling blocks towards convergence must be removed. Of these, experience has shown that numerical errors can potentially cripple the ability to reliably obtain solutions due to an errant calculation of the search direction. One of the most significant contributors to the build-up of numerical error is in the calculation of the Jacobian. The Jacobian (or gradient matrix) is used in every functional non-linear solver (aside from perhaps swarming or evolutionary algorithms) to calculate the search direction at each step. The easiest and most straightforward way to calculate the gradient is to take advantage of finite differencing, a technique that approximates the gradient through multiple function evaluations. There are many finite difference methods available, but all contribute some level of numerical error. This damages the ultimate aim of robust convergence.

As a result, the TEI algorithm eliminates a significant portion of the numerical error by using analytic derivatives, derived specifically for the TEI problem. An additional benefit to providing fully analytic gradients is run time. The accuracy in finite difference methods is directly related to number of function calls, with each function call doubling the cost of propagation. The derivatives are analytic in the sense that an equation can be derived for all relationships. However, the types of derivatives are divided into two categories: exact and state transition matrix (STM) based.

\section{A. Exact Derivatives}

Exact derivatives are exact in the sense that an equation for the gradient can be obtained analytically, and the resultant equation is a function of known quantities. This encompasses the vast majority of the derivative functions. For the TEI problem, all of the objective gradients are exact, which is simply the cost of all of the burns. In addition, a majority of the constraint gradients are also exact. These include time equality and inequality constraints, EI parameter constraints latitude, longitude, etc and the target line function constraint of the EI parameters. Also, the apolune constraint at the second burn is exact in all control parameters. The perilune constraints at the second and third burns respectively have portions that are exact and portions that are not. Basically, any constraint to parameter relationship that is not dependent on propagated time is exact. Thus, the perilune constraint with respect to the state at the burn is exact, but with respect to a previous time such as the time of the first burn, it is not exact. Finally, when slack variables are used to transform inequality constraints into equality constraints, these functions are also exact. The partial derivatives of these functions vary in complexity but are known.

\section{B. State Transition Matrix (STM) Based Derivatives}

State Transition Matrix based gradients are obtained from partial derivative equations with linear assumptions and require propagated data in order to be calculated. The use of STM for gradient calculation is well established ${ }^{5}$. The STM method uses linear approximations of state perturbations. The use of these assumptions is collectively known as linear perturbation theory. This theory posits that if the state is perturbed along a path at some time along that path this variation can be related to a corresponding variation in the initial state at the initial time by use of the following equation:

$$
\delta \vec{x}(t)=\Phi\left(t, t_{0}\right) \delta \vec{x}\left(t_{0}\right)
$$

Here the matrix refers to the state transition matrix which is obtained by integrating the state propagation matrix along with the equations of motion. The state propagation matrix is the partial derivative of the time derivative of the state equation vector with respect to the state vector. Another key relation is between the differential and the time independent variation:

$$
d(\vec{x})=\delta(\vec{x})+\dot{\bar{x}} d t
$$

This relationship enables calculation of a partial derivative with respect to a time other than the time associated with the state vector $\mathrm{x}$.

For the TEI problem the primary constraint requiring the use of state transition matrices is the sphere of influence state constraint that requires the forward propagating arc from the Moon and the backwards propagated arc from the Earth to match in position and velocity. In addition the perilune constraints at burns two and three with respect to 
burn times prior also require use of the STM. It is unsurprising that these constraints are the most difficult to meet. Even with the use of STM, the linear assumptions contribute to an imprecise calculation of the search direction.

\section{Keys to Problem Convergence}

The desire for a flexible autonomous algorithm is naturally coupled with a need to minimize excessive iteration. Once the analytic guess is determined, it is vital that the targeter quickly converge to a feasible, flyable solution. Unfortunately, there are no known criteria for the TEI problem from which convergence can be guaranteed. The following set of topics summarizes some of the most important tools that the targeting algorithm uses to ensure convergence, verified solely with testing (see Section VII).

\section{A. Lowering the Initial $\Delta \mathbf{V}$ Estimate}

As described in Section II, the initial guess is a critical component in the solution finding process. If the initial guess is not in the neighborhood of the final solution, it is impossible to guarantee convergence. Unfortunately it is difficult to measure the degree to which an initial guess meets this criterion and impossible to prove analytically. Thus, improvements found in the initial guess design are extremely beneficial.

One of the most important attributes of the initial guess is the sequence cost, the sum of the $\Delta \mathrm{v}$ costs . Regardless of the formulation, optimal or feasible, the cost of the maneuver sequence plays a pivotal role in determining the amount of iteration necessary to obtain a solution. In the original formulation for the three burn sequence, the second maneuver was assumed to occur at the apolune of the first transfer ellipse. In actuality, the optimal solutions do not occur at this point, but generally at some delta true anomaly from apolune. Likewise, in the optimal solution, the first and second transfer ellipses tend to have almost equal eccentricities. Since the assumption to apply the burn at apolune is not required to have a viable initial guess, the formulation has the potential to be modified. The following derivation is a closed form solution where the eccentricities of the two transfer ellipses are made equal.

To begin, the unit vector associated with the second burn must be in the plane of all the orbits. This is bounded by the following equation, developed in the original formulation:

$$
\hat{r}_{\text {burn } 2}=\frac{\left|\hat{r}_{a 2} \times\left(\hat{h}_{3} \times \hat{h}_{0}\right)\right| \times \hat{h}_{0}}{\left.\mid \hat{r}_{a 2} \times\left(\hat{h}_{3} \times \hat{h}_{0}\right)\right] \times \hat{h}_{0} \mid}
$$

In this equation, the integer subscripts specify the orbit number increasing from the parking orbit (0) to the first and second transfer ellipses $(1,2)$ to the departure hyperbola (3). Originally, the orbit was defined by making this vector the same as the apolune of the first transfer ellipse:

$$
\hat{r}_{a 1}=\hat{r}_{\text {burn } 2}
$$

Since the optimal occurs at a different true anomaly, a new equation with an additional control parameter can be written:

$$
\hat{r}_{a 1}=\hat{r}_{\text {burn } 2} \cos \left(180^{\circ}-v_{\text {burn } 21}\right)+\left[\hat{h}_{1} \times \hat{r}_{\text {burn } 2}\right\rfloor \sin \left(180^{\circ}-v_{\text {burn } 21}\right)
$$

Here the true anomaly of the second burn along the first transfer ellipse is an unknown. But a constraint can be added, where the eccentricities or the transfer ellipses are set to be equal. Additional equations can be written; the position vector of the second burn is now an unknown, dependent on the true anomaly according to the following equation:

$$
r_{\text {burn } 2}=\frac{r_{a 1}\left(1-e_{1}\right)}{1+e_{1} \cos \left(v_{b u r n 21}\right)}
$$

Likewise, the second transfer ellipse remains undefined, dependent on the unit vector of the apolune of the second transfer ellipse, which is constrained to be along the same line of apsides as the departure hyperbola perilune, 
specified by the target $\mathrm{v}_{\infty}$ vector along with the magnitude of the position vector of the second burn. The eccentricity of the second ellipse is defined in the following equation:

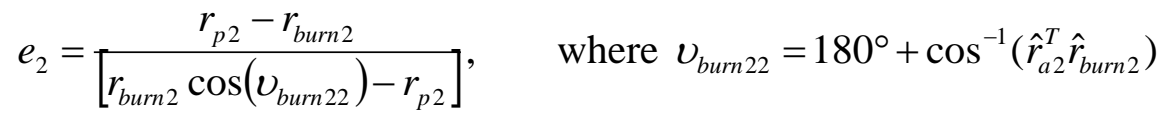

Combining equations 6 and 7, the eccentricity of the second transfer ellipse is now just a function of the eccentricity of the first transfer ellipse and the true anomaly of burn two along the first transfer ellipse:

$$
e_{2}=\frac{r_{p 2}-\left[\frac{r_{a 1}\left(1-e_{1}\right)}{1+e_{1} \cos \left(v_{b u r n 21}\right)}\right]}{\left[\left[\frac{r_{a 1}\left(1-e_{1}\right)}{1+e_{1} \cos \left(v_{b u r n 21}\right)}\right]\left[-\hat{r}_{a 2}^{T} \hat{r}_{b u r n 2}\right]-r_{p 2}\right]}
$$

At this point, the assumption of the equivalency of the eccentricities of the orbits can be applied:

$$
e_{2}=e_{1}
$$

With this assumption there are still three unknowns and only two equations. An additional relation is needed. The eccentricity of the first transfer ellipse is known if the moon parking orbit is circular, since the apolune of the first transfer ellipse is specified, and the perilune must be equal to the parking orbit radius:

$$
e_{1}=\frac{r_{a 1}-r_{p 1}}{r_{a 1}+r_{p 1}}
$$

If the parking orbit is eccentric, the value of is unknown and as a result, so is the eccentricity of the transfer ellipse. However, an additional assumption can be applied for near circular parking orbits where:

$$
r_{p 1}=r_{p 0}
$$

With the eccentricity of the first transfer ellipse known equations 8 and 9 can be arranged and solved for the true anomaly of burn two along the first transfer ellipse:

$$
v_{\text {burn } 21}=\cos ^{-1}\left[\frac{\left[e_{1}^{2} r_{a 1}\left(\hat{r}_{a 2}^{T} \hat{r}_{\text {burn } 2}\right)-e_{1}\left(r_{a 1} \hat{r}_{a 2}^{T} \hat{r}_{b u r n 2}\right)+r_{p 2}-r_{a 1}\right]+\left(r_{a 1}-r_{p 2}\right)}{e_{1}^{2} r_{p 2}+e_{1} r_{p 2}}\right]
$$

All orbits and ignition times are now defined. The efficacy of this assumption change is immediately apparent with an average drop of about $250 \mathrm{~m} / \mathrm{s}$ in $\Delta \mathrm{v}$ cost, without any sacrifice in accuracy. Since a large portion of the initial iterates are used to bring down the impulsive cost, this change is a significant aid to achieving successful convergence on flyable trajectories. Clearly more work in this area, primarily in the design of the transfer ellipses, could move the initial guess even closer to the optimal solution. However, additional improvements could come at the cost of greater complexity. Some empirical approximations are discussed below, but ideally analytic expressions would be derived.

\section{B. Backwards Propagation from Earth}

Targeting a fixed Moon-based hyperbolic $\mathrm{v}_{\infty}$ vector is very different from successfully reaching a specific EI state. Over a multiple day transfer the impact of Earth and Sun gravitational perturbations can invoke large changes in the 
final state at the Earth leading the spacecraft far from the desired EI targets. In order to obtain a flyable trajectory to Earth the multi-body trajectory must be iterated on with a fully propagated Moon-Earth arc.

Instead of propagating forward to EI, the optimization parameters associated with the target Earth state are propagated backwards in time. The alternative is to propagate the state forward from the final TEI maneuver all the way to EI. However, any change in the state at the Moon is magnified the nearer the trajectory gets to Earth. Setting a single point to match position and velocity for a continuous trajectory near the boundary of the Moon sphere of influence (SOI) reduces the convergence difficulties associated with the large swings in EI parameters that correspond to small changes near the Moon. Of the keys to convergence, this is the most significant.

\section{Estimating Optimal Latitude}

Section II described how a combination radial magnitude/latitude target set is use to formulate an estimate for the $\mathrm{v}_{\infty}$ vector. Up to this point, this latitude target was a freely specified input. But as discussed in detail with regards to the EI constraints, the optimal EI latitude is closely related to the latitude of the antipode. Since the geometry is known apriori analytically, this relationship could be taken advantage of in the initial guess procedure. While a more analytic approach is still in the works, an empirical estimation appears to be effective. Figure 2 demonstrates the tight relationship:

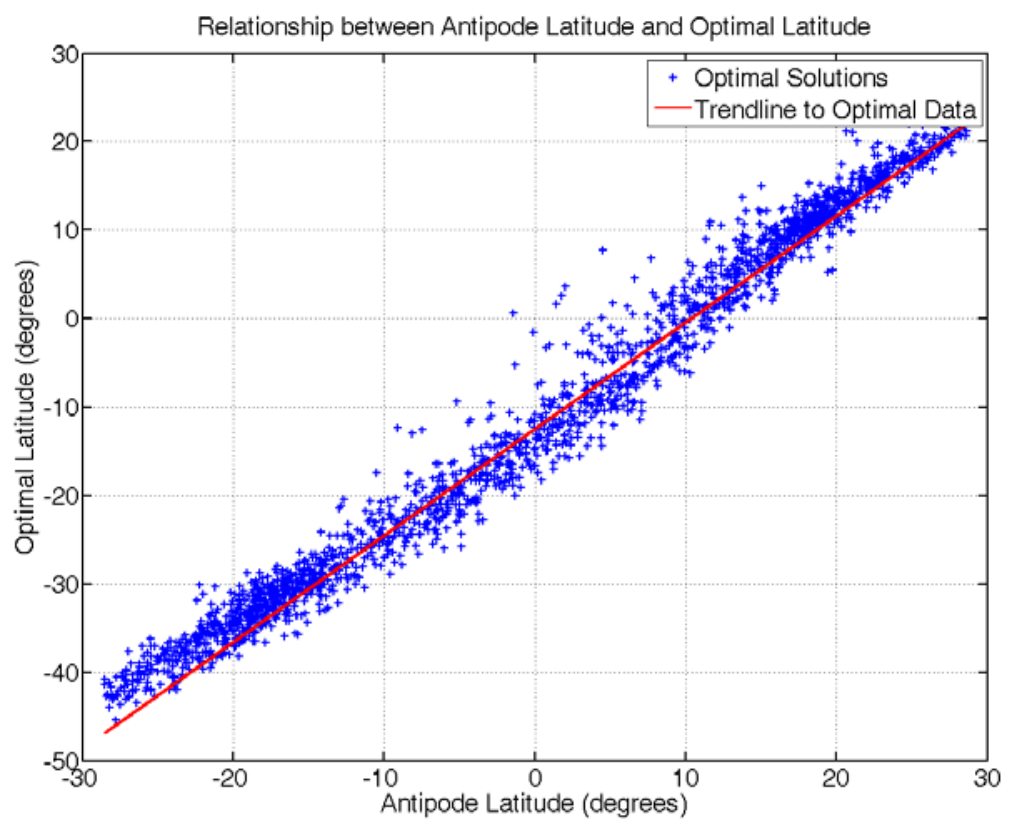

Figure 2. Linear relationship between antipode and optimal latitude.

Like the improvement in the initial estimate for the impulsive $\Delta v$ cost, this estimate reduces iteration by providing a more accurate $\mathrm{v}_{\infty}$ target. More research into this relationship will enhance the capability, as this particular slope/intercept set is correlated with the EI target lines associated with a San Clemente landing.

\section{Second Local Optimum Considerations}

In the process of exploring the Earth/Moon trajectory space, it has become clear that multiple locally optimal solutions exist ${ }^{4}$. The size of the trade space is determined in part by the constraints. But more fundamental is the geometry itself. There are many feasible, flyable TEI solutions that target the same $\mathrm{v}_{\infty}$ vector, but it would still make sense that only one geometry would be optimal. One of the first steps in the initial guess process is the definition of the angular momentum vector for the departure hyperbola. The equation for this vector is written such that plane change with respect to the initial parking orbit is smallest, a true property of the optimal solution. However, the equation, given below, does not account for Earth and Sun perturbation effects or EI target constraints, but simply a single gravitational body:

$$
\hat{h}_{f}=\frac{\hat{v}_{\infty}^{+} \times\left(\hat{h}_{0} \times \hat{v}_{\infty}^{+}\right)}{\mid \hat{v}_{\infty}^{+} \times\left(\hat{h}_{0} \times \hat{v}_{\infty}^{+}\right)}
$$


Thus, when the plane change gets close to $90^{\circ}$ the unknown perturbations can cause the actual plane change in a full model solution to be greater than $90^{\circ}$ when it appeared the trajectory would require less. In that instance, it can be immediately confirmed that this is not the true optimal. So while in fact the cost has been minimized with respect to the local feasible solutions, the entire trade space has not been examined. In order to do this, a new initial guess can be generated where the angular momentum vector is in the exact opposite direction. The same $\mathrm{v}_{\infty}$ vector is being targeted, but the entire sequence is rotated $180^{\circ}$ around the Moon as shown in Figure 3. This initial guess will also have a plane change close to $90^{\circ}$, and likely larger than $90^{\circ}$. But in this instance the perturbation effects bring down the amount of total plane change conducted in the final solution and thus is truly optimal. Without advanced knowledge of the perturbation and EI constraint effects both the first and second local optimum solutions should be evaluated.

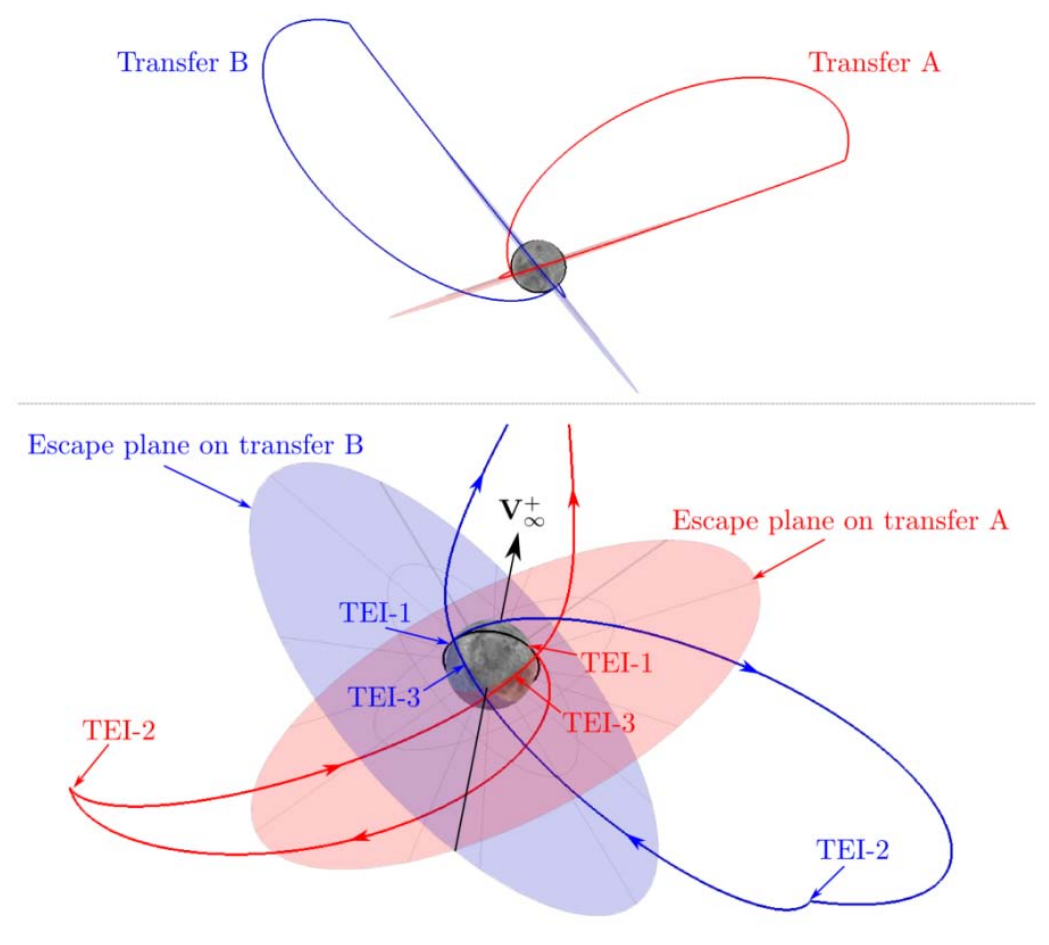

Figure 3. Two locally optimal solutions to the three-burn TEI problem. ${ }^{5}$

\section{E. Margin on Apolune and Perilune Constraints}

It is difficult to guarantee convergence for trajectory optimization problems due to the non-linear nature of the trade space and the inability for optimizers to navigate between local optimums without stumbling through infeasibilities that can lead the optimizer to singularities and crash the program. Thus, it is extremely important to set up the problem so that the optimizer or other non-linear correcting algorithm has a simple job to do: follow the path of least resistance all the way to the optimal while avoiding the ping-pong effect of boundary constraint bumpers. The effect can occur if the objective function contours pull the solution along the constraint boundary and the initial guess is placed along one of these boundaries. The TEI problem possesses many undesirable constraints. The two most important constraints are the apolune and perilune limits. The apolune constraint prevents the solution from approaching parabolic at the second maneuver, which is in fact the true $\Delta \mathrm{v}$ optimal, and the perilune constraint prevents a second unplanned Moon landing from occurring. If the initial guess was designed based strictly on these boundaries, a non-linear iterator may experience limited or low success finding a feasible solution. But if margin is given to these constraints, the curvature of the objective function contours will funnel the consecutive iterates much more readily to the optimal in a predictable, robust manner. In the results published in this document, a $10 \%$ margin was applied to these constraints, which appears to be adequate for successful convergence. 


\section{Search Algorithm Independence}

There exists a variety of algorithms that take advantage of the assumption that the solution lies within the vicinity of the initial guess by designing search directions for the control parameters that point towards the minimum feasible solution as efficiently as possible. The TEI autonomous targeter takes advantage of two of these techniques. One option is a full optimization mode, where the constraints are set to 0 and total fuel consumption objective function is simultaneously minimized. A Sequential Quadratic Program (SQP) is selected as the algorithm for full optimization due to the quadratic nature of the solution space where the initial guess is in the vicinity of the solution. The second option is a minimum norm mode. The minimum norm algorithm does not attempt to minimize the solution but instead chooses a search direction that minimizes the constraint error. This correction is calculated using the simple pseudo-inverse mathematical operation. Without a $\Delta \mathrm{v}$ constraint, this approach always converges robustly close to the fuel cost of the initial guess. If a mission cost constraint is added in the form of a maximum quantity of fuel ( limit), convergence can still be robust, but careful attention must be made to the constraint value used. The optimal value for a given epoch and geometry is not known apriori, and if the limit is too constraining, the algorithm could be set up to solve a problem in which no solution exists. As long as the propellant load exceeds performance estimates, this value could be set to this propellant limit with expectation of convergence. If an optimizer is not the ideal fit for an onboard algorithm, this is a suitable option for Orion.

\section{Single Maneuver Finite Burn Conversion}

Once a converged impulsive solution has been obtained, the targeting algorithm can convert each individual $\Delta \mathrm{v}$ impulse into a finite burn command. If the time of the impulsive is , then the finite burn maneuver begins at and ends at . The optimization variables are (the time from to ) and (the time from

to ). The initial state of the finite burn inherits the state on the impulsive trajectory at . The maneuver control variables are the right ascension and declination of the thrust vector, and their time derivatives and . A state continuity constraint (both position and velocity) is imposed that the final state of the finite burn (at ) is equal to the state at the same time on the impulsive trajectory. The direction is used as an initial guess for the thrust direction. The rocket equation provides an estimate of the burn time:

$$
\Delta t_{1}=\Delta t_{2}=\frac{1}{2}\left[\frac{c \cdot m_{0}}{T}\left(1-e^{-\Delta v / c}\right)\right]
$$

Where $\mathrm{c}$ is the engine Isp and $\mathrm{T}$ is the engine thrust. Thus the problem is a two point boundary value problem where the number of controls and constraints are equal (in this case, six of each), and a simple non-linear solver may be used. The minimum norm algorithm converges these individual finite burns very quickly and robustly, even for low thrust engines. The resultant solution is a feasible finite burn fit to the initial impulsive trajectory.

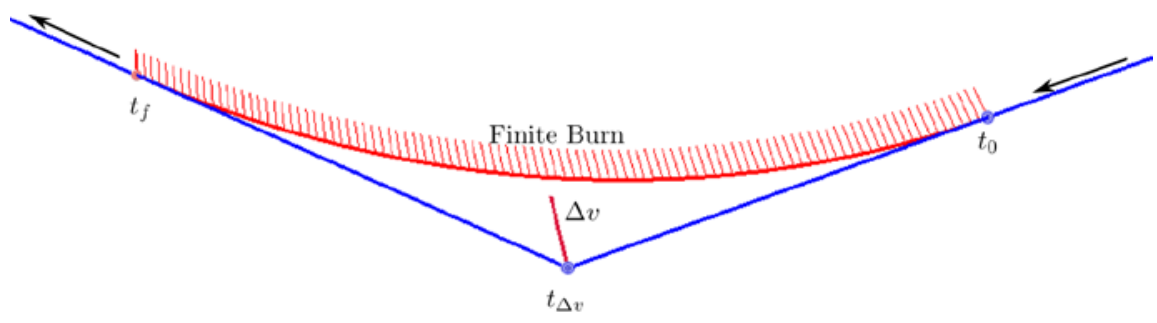

Figure 4. Finite burn conversion.

A feasible finite burn is fit to a trajectory containing an impulsive $\Delta \mathrm{v}$. 


\section{Monte Carlo Results}

As discussed in Sections II and III, convergence cannot be guaranteed for the non-linear multi-body TEI problem. Thus, the merit of this or any other algorithm can only be established through rigorous testing. To that end, a random set of Monte Carlo cases is designed in order to assess algorithm performance. Since algorithms can be tweaked to perform well on specific outliers, the final Monte Carlo set of random conditions was selected after the version of the algorithm was fixed and was not rerun with subsequent changes.

As Orion is designed to depart from a $100 \mathrm{~km}$ retrograde circular Moon orbit at any time from any orbit orientation, the variables in the Monte Carlo are dispersed as follows:

Table 1. Monte Carlo Dispersion Table

\begin{tabular}{|l|l|l|l|}
\hline Variable & Distribution & Minimum Value & Maximum Value \\
\hline Epoch & Uniform & 2009 calendar yr & 2039 calendar yr \\
\hline Inclination & Uniform & 85 degrees & 180 degrees \\
\hline Right Ascension of Ascending Node & Uniform & 0 degrees & 360 degrees \\
\hline
\end{tabular}

Eccentricity, semi-major axis, and argument of periapsis were not dispersed as these parameters have known quantities or in the case of the argument of periapsis, are undefined. The epoch bounds were established to encompass a full 19 year Lunar nodal cycle. 2000 uniformly dispersed cases were drawn randomly for each parameter.

Two search algorithms were tested, one optimizer and one minimum norm algorithm. The optimizer is the familiar Harwell Subroutine Library subroutine VF13AD, a sequential quadratic programming (SQP) based optimizer. SQP algorithms are equipped to handle non-linear orbital problems due to the quadratic nature of the solution space near the optimal. The use of the SQP was tuned to the TEI problem by applying a step size limiter on each control parameter. The minimum norm routine is the Moore-Penrose pseudoinverse algorithm (referred to as PINV in this document). The pseudoinverse uses LU decomposition on a non-square matrix to calculate the minimum norm correction vector. The pseudoinverse is often used to calculate the least squares solution for a system of linear equations with multiple solutions where the number of variables exceeds the number of equations. Similarly, the pseudoinverse is used to find the nearest solution to the initial guess in the TEI problem where the number of control parameters exceeds the number of constraints. The step size for each PINV iterate is limited to prevent divergence; in this instance the step size is modulated by the magnitude of the constraint error.

In order to apply the search algorithms appropriately, the objective function must vary between the optimizer and the minimum norm algorithm. In the case of the optimizer the objective function for the impulsive portion of the problem is the sum of the three individual $\Delta \mathrm{v}$ 's. For the PINV algorithm, this objective function cann ot exist and is replaced with a constraint on the same sum. If this constraint is eliminated, the solution closest to the initial guess is obtained which has a good chance of exceeding Orion's propellant load. However if the constraint is too low, the solution space could be empty. As a result, careful attention must be placed on this constraint limit.

Two different examples of assessing to a $\Delta \mathrm{v}$ limit are demonstrated. The first is a constant limit for all Monte Carlo cases, set as a maximum value of $1.3 \mathrm{~km} / \mathrm{s}$. This limit is based on extensive performance analysis by the Orion team $^{1}$ and with the total TEI $\mathrm{v}$ represents a small amount of margin applied to the worst TEI case expected for Orion, at $1.26 \mathrm{~km} / \mathrm{s}$. Ideally, the full amount of propellant to ensure full temporal and surface coverage would be loaded on the vehicle. The second example of a $\Delta \mathrm{v}$ constraint is an Orion reduced propellant limit curve. The curve used in this example is a real expectation of the propellant currently planned for Orion and represents a conservative estimate of what should be available after lunar operations are complete and the crew is ready to return to Earth. While the one applied in this example is a function of Moon Orbit inclination, any line could be investigated. This line immediately eliminates certain mission scenarios. Thus, the non-executable maneuvers (those optimal cases above the propellant limit) are removed. In the end, it is possible that the propellant load will be very close to the optimal return cost, so it is a profitable exercise to see how successful PINV achieves convergence when a limit is set very close to the optimal value. This becomes increasingly difficult as the number of solutions approaches one, the optimal.

Note that for both VF13AD and PINV, the constraints are all equality constraints set to zero, which is necessary for the PINV algorithm. Those constraints that have inequality bounds are converted into equality constraints with the use of slack variables.

Figure 5 demonstrates the results of the 2000 case Monte Carlos. For the VF13AD optimizer and $1.3 \mathrm{~km} / \mathrm{s}$ feasible constrained PINV algorithms, all 2000 cases converged into flyable finite burn solutions to acceptable tolerances. Figure 5(a) shows the reduction in cost that transpires through iteration from the initial guess to the final optimal solution. In contrast, Fig. 5(b) shows that a minimum constraint results in two families of solutions, clearly demarcated by the constraint line. Figures 5(c) and 5(d) show that the finite burn solution is the feasible equivalent 
of the impulsive solution for both the optimal and feasible algorithms. In each one of these cases, the spacecraft can perform the finite burn maneuvers as designed; there are no approximations or linear assumptions to caveat.

It is important to note the speed of the algorithm. The vast majority of the run time occurs in the impulsive phase. While the optimizer averages 855 iterations for the impulsive phase, the average run time for the whole program is less than a minute (52 seconds) and never exceeds 3 minutes. Of course run times depend on machine speed, but the run time is mentioned here to provide an indication of the ease in which optimal solutions could be obtained realtime. The run time for PINV and the $1.3 \mathrm{~km}$ per second limit is similar, with an average of 59 seconds. Most of the cases for PINV are obtained faster, but there are also more outliers biasing the average with a maximum run time of 4.6 minutes. The speed of obtaining a PINV solution is dependent on the step size limitation formulation and how close the constraint is to the optimal. The step size limitation could be relaxed but would then be more susceptible to divergence especially with a tight constraint boundary.

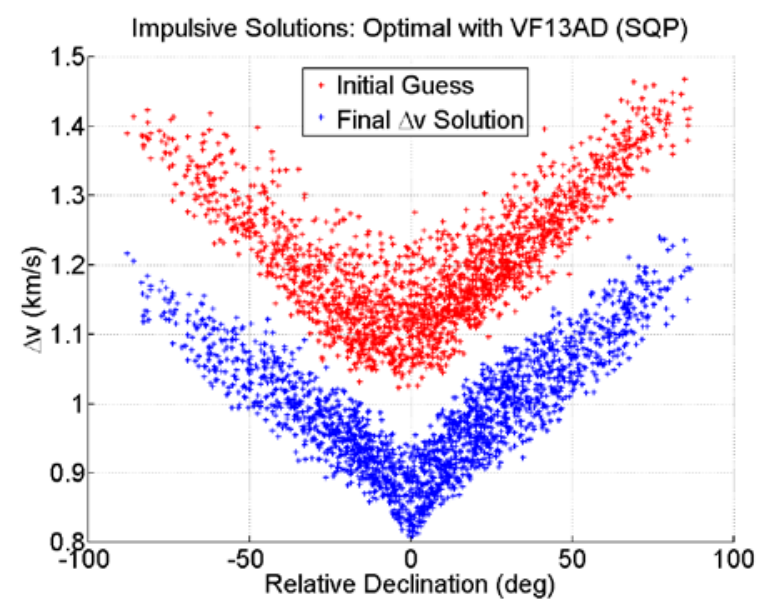

(a) VF13AD Impulsive Results

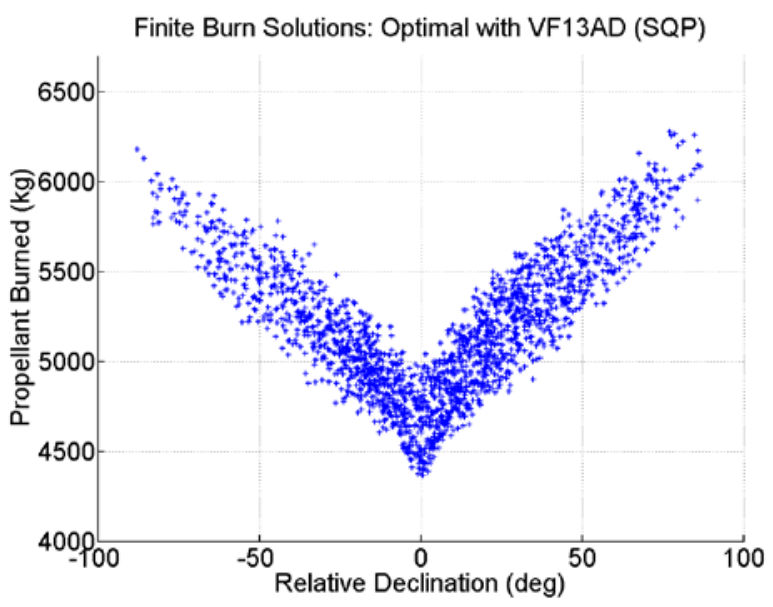

(c) VF13AD Finite Burn Results

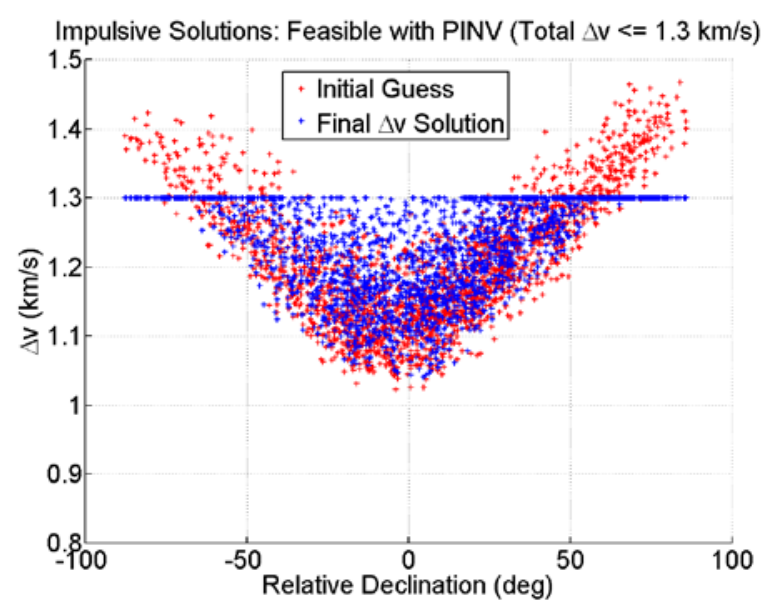

(b) PINV Impulsive Results

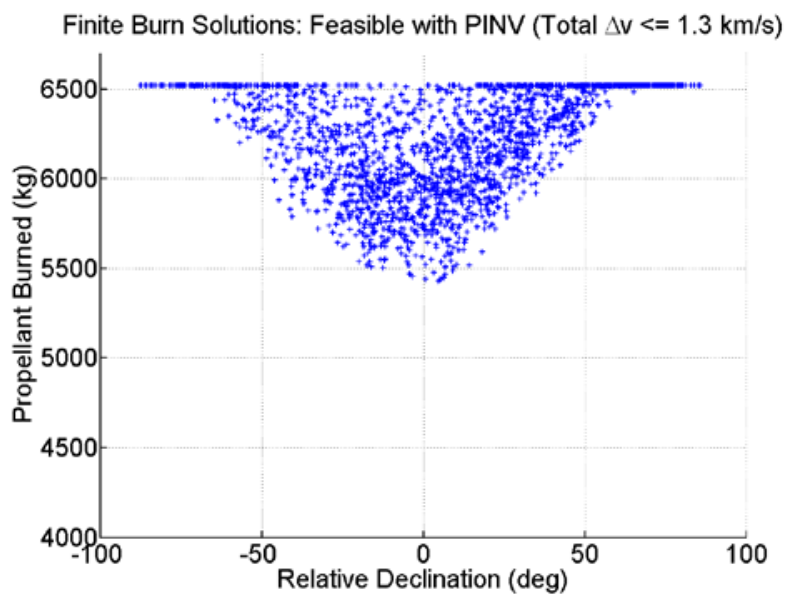

(d) PINV Finite Burn Results

Figure 5. Impulsive and finite burn results for VF13AD and PINV with $1.3 \mathrm{~km} / \mathrm{s}$ constraint

Figure 6 shows the final Entry Interface (EI) conditions for all of the optimal and $1.3 \mathrm{~km} / \mathrm{s}$ PINV constrained cases. The blue arrows represent the azimuth of the incoming spacecraft. In all 4000 cases the algorithm obtained an EI condition targeting the nominal San Clemente landing site. In other words, even in an abort situation, the TEI targeter can bring the spacecraft back to San Clemente every time. Of course, down modes are available that provide much easier targets, such as an EI condition that is comprised of only an altitude and flight path angle constraint. 
However in the instances shown here, a representative set of all cases with a reasonable constraint boundary, this down mode is unnecessary.

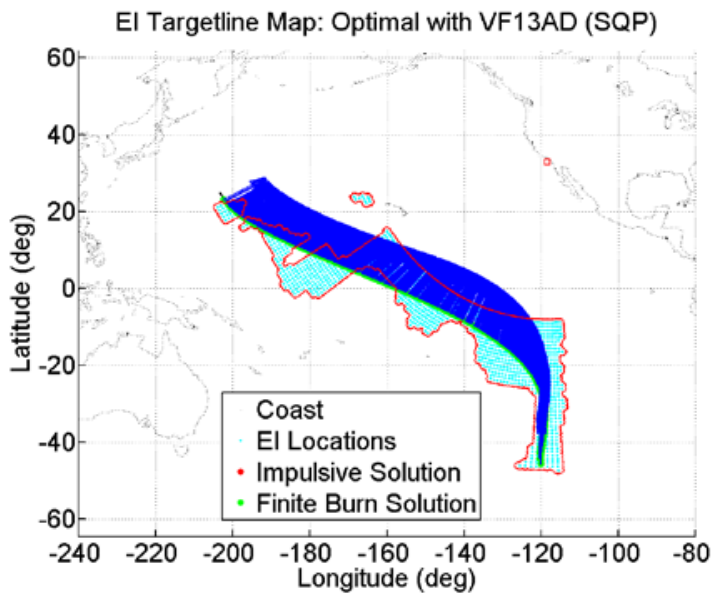

(a) VF13AD EI Target Line Results

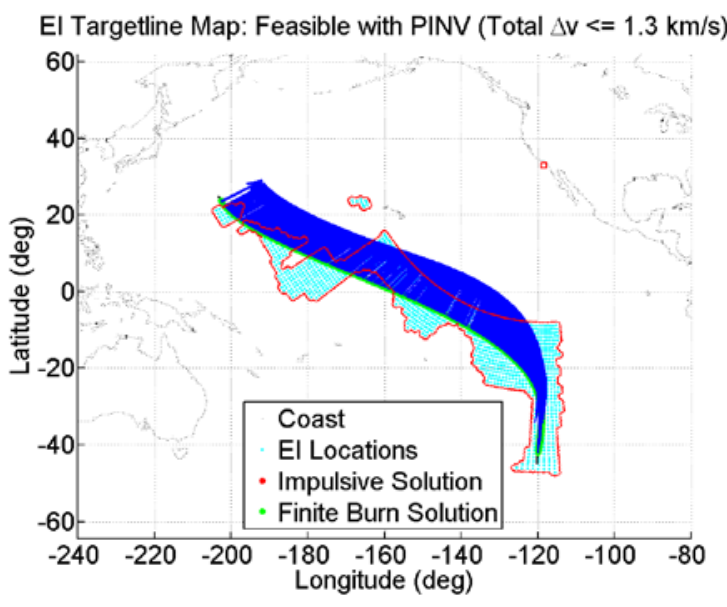

(b) PINV EI Target Line Results

\section{Figure 6. EI Target Line Results}

The constraint line is another way to represent the application of a propellant load limit. The left hand plot in Figure 7(a) shows that with the current estimate, 3.6\% of the random cases in this study are not executable. With or without the targeter, Orion would be stuck in lunar orbit in these scenarios. Figure 7(b) shows how the targeter performs for the rest of the cases. In both figures, the blue points represent the VF13AD optimal impulsive solutions, and in Fig. 7(b) the red points indicate only those cases where PINV could not obtain a solution below the boundary. As expected a few cases fail to converge and all are close to the constraint boundary. However, the percentage of cases that do fail is small, less than $1 \%$ of the total executable. These facts demonstrate the limitation of the PINV search algorithm, not the targeter itself. The optimizer produces more intelligent search directions than the PINV algorithm which is not an optimizer but is being forced to act like one. Overall, it makes much more sense to use an optimizer in the situation when the propellant load is close to the optimal.

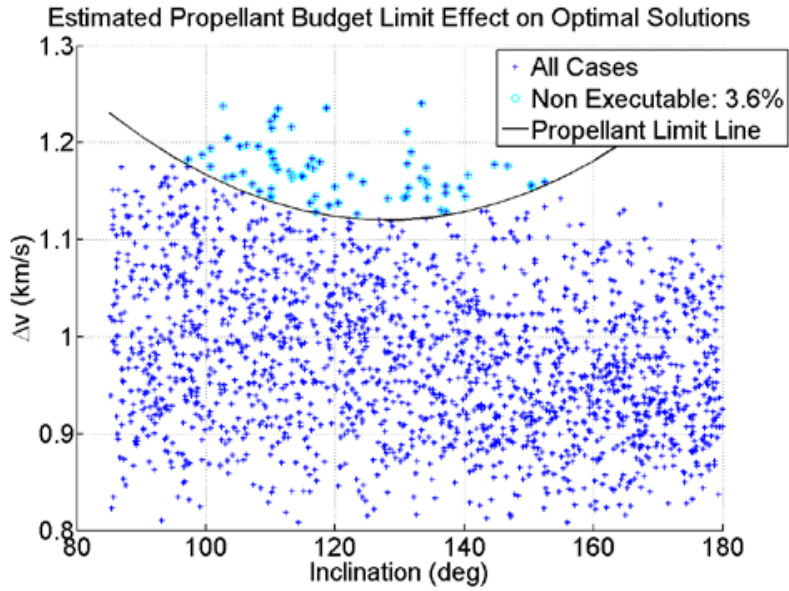

(a) Lunar surface and temporal coverage limitations

Figure 7. Propellant limit curve results

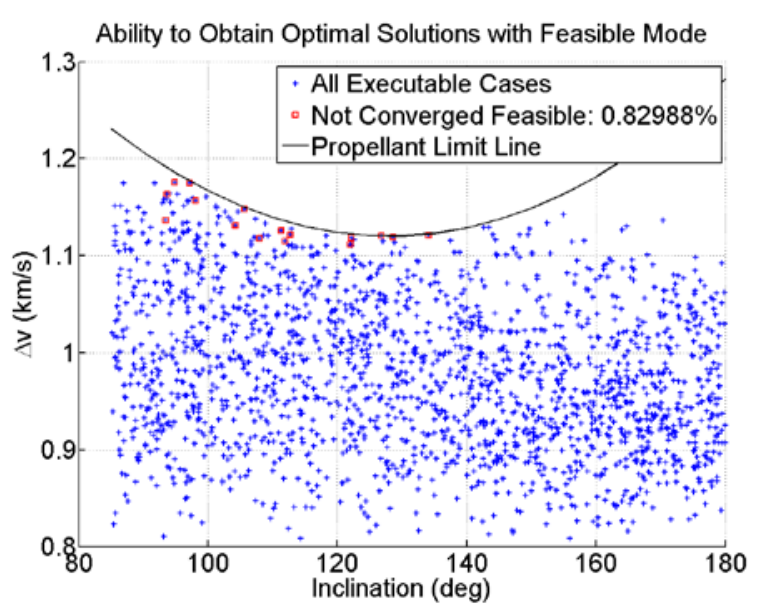

(b) Using PINV to obtain near optimal solutions 


\section{Conclusion}

The Trans-Earth Injection (TEI) problem is not a simple one. There are no fully analytic solutions to the problem, especially when considering third body perturbations, tightly coupled Entry Interface constraints and patched conics. But by applying the known analytics and an understanding of the TEI solution space along with a tightly constrained search algorithm to the problem, a robust algorithm emerges. The framework for a multi-maneuver TEI sequence algorithm is now verifiably autonomous.

\section{References}

${ }^{1}$ Williams, J., Davis, E. C., Lee, D. E., Condon, G. L., Dawn, T. F., and Qu, M. “Global Performance Characterization of the Three Burn Trans-Earth Injection Maneuver Sequence over the Lunar Nodal Cycle”, AAS 09-380, 2009.

${ }^{2}$ Saudemont, R. R. and Cesar A. Ocampo. "Initial Trajectory Model for a Multi-Maneuver Moon to Earth Abort Sequence," AAS Space Flight Mechanics Meeting, 2009.

${ }^{3}$ Edelbaum, T. N. “Optimal Nonplanar Escape from Circular Orbits,” AIAA Journal 70-1038, 1970.

${ }^{4}$ Senent, J. S. “An Optimal Initial Guess Generator for Entry Interface Targeters,” AAS 09-426, 2009.

${ }^{5}$ Whitley, R. J. “Direct Multiple Shooting Optimization with Variable Problem Parameters”, $47^{\text {th }}$ AIAA Aerospace Sciences Meeting. AIAA-2009-803, 2009.

${ }^{6}$ Zimmer, S. and Cesar A. Ocampo. “Analytical Gradients for Gravity Assist Trajectories Using Constant Specific Impulse Engines”, Journal of Guidance, Control, and Dynamics, Vol.28, No.4, 2005.

${ }^{7}$ Williams, J., Stewart, S. M., Condon, G. L., Lee, D. E., Davis, E. C., Senent, J. S., and Dawn, T. F. “The Mission Assessment Post Processor (MAPP): A New Tool for Performance Evaluation of Human Lunar Missions”, AAS 10-191, 2010.

${ }^{8}$ Robinson, S. and David Geller. “A Simple Targeting Procedure for Lunar Trans-Earth Injection”, AIAA Guidance, Navigation and Control Conference, AIAA 2009-6107, 2009.

${ }^{9}$ Scaritt, S. Marchand, B. G., and Michael W. Weeks. “An Autonomous Onboard Targeting Algorithm Using Finite Trust Maneuvers.” AIAA Guidance, Navigation and Control Conference, AIAA 2009-6104, 2009. 